\title{
The potential impact of the hypovitaminosis D on metabolic complications in obese adolescents - Preliminary results
}

\author{
Malgorzata Wojcik', Dominika Janus' ${ }^{1}$ Anna Kalicka-Kasperczyk¹, Krystyna Sztefko², \\ Jerzy B. Starzyk ${ }^{1}$ \\ 1 Department of Pediatric and Adolescent Endocrinology, Chair of Pediatrics, Institute of Pediatrics, Jagiellonian \\ University Medical College, Krakow, Poland \\ ${ }^{2}$ Department of Clinical Biochemistry, Institute of Pediatrics, Jagiellonian University Medical College, Krakow, Poland
}

Wojcik M, Janus D, Kalicka-Kasperczyk A, Sztefko K, Starzyk J B. The potential impact of the hypovitaminosis D on metabolic complications in obese adolescents - Preliminary results. Ann Agric Environ Med. 2017; 24(4): 636-639. doi: 10.5604/12321966.1230676

\begin{abstract}
Introduction and objective. Vitamin D deficiency is common in obesity; however, its contribution in the development of metabolic complications remains uncertain. The aim of the study was to examine the relationships between vitamin $D$ status and metabolic complications.

Materials and method. The results of blood pressure measurements, biochemical tests and ultrasound of the liver were compared in both groups. The study was conducted at the Children's University Hospital in Krakow, Poland. 30 obese adolescents (mean 13.23y.o.); 18 with $250 \mathrm{HD}$ levels $<20 \mathrm{ng} / \mathrm{mL}, 12$ with $25 \mathrm{OHD}>20 \mathrm{ng} / \mathrm{mL}$.

Results. The vitamin D deficient group presented with significantly higher values of the diastolic blood pressure (125.9vs.115 mmHg), uric acid level (384.7vs.301.5umol/L) and lower phosphorus level (1.4vs.1.65mmol/L), higher prevalence of arterial hypertension (44vs.8.3\%), and liver steatosis (25vs.8.3\%); lower, but not significantly, levels of fibroblast growth factor 23 and fibroblast growth factor 19.

Conclusions. Hypovitaminosis D in obese adolescents is associated with higher prevalence of arterial hypertension, liver steatosis, elevated serum uric acid and low phosphorus levels. The potential contribution of the fibroblast growth factor 23 and fibroblast growth factor 19 in these complications development needs further investigation.
\end{abstract}

\section{Key words}

obesity, hypovitaminosis D, uric acid, arterial hypertension, fibroblast growth factor 23, fibroblast growth factor 19 , adolescents

\section{INTRODUCTION}

The global escalation of childhood obesity is a major public health issue, as excessive adiposity is the leading cause of metabolic and cardiovascular disease and their related mortality in adulthood. The main causes of this increase in cardiometabolic risk are a decrease in insulin sensitivity and compensatory hyperinsulinemia [1, 2, 3, 4]. A strong association between insulin resistance and an excess of fat tissue has been recognized for decades, but some details of its origin remain unclear to date. Undoubtedly there is a strong correlation between genetic background and environmental factors. Since it is not yet possible to modify the genetic background, it is essential to identify modifiable environmental factors. One of the most discussed in literature possible contributors of the obesity itself and its complications remains hypovitaminosis D. Many studies confirmed a high prevalence of vitamin D deficiency in obese patients, since both conditions are often associated. Approximately $60 \%$ of children and adolescents are overweight or obese in comparison to $20 \%$ of those who are non-obese subjects $[1,2,3,4]$. However, an inverse correlation between body mass index (BMI), fat tissue content and vitamin D levels

Address for correspondence: Malgorzata Wojcik, Department of Pediatric and Adolescent Endocrinology, Chair of Pediatrics, Pediatric Institute, Jagiellonian University Medical College, Krakow, Poland

E-mail: malgorzata.wojcik@uj.edu.pl

Received: 1 March 2014; accepted: 10 October 2014; first published on January, 2017 had been proved in many studies, and a recently published metaanalysis of 21 adult cohorts (up to 42,024 participants) revealed that higher a BMI leads to a lower vitamin D level. However, low vitamin D level does not contribute to an increase in the BMI [3]. These results confirm that hypovitaminosis $\mathrm{D}$ is rather a consequence, not a cause of obesity. From this point of view, low vitamin D may not be considered as an isolated condition, but rather the potential indicator of the risk of other metabolic complications in obese patients. Moreover, since puberty is a critical life-stage period characterized by rapid growth and development, the deficiency of vitamin D may be relative and difficult to diagnose, because there are no specific reference values and cut-off points for vitamin D level in adolescents. Recent studies had shown, that hypovitaminosis $\mathrm{D}$ might be not only one of the many obesity complications, but may be also a potential trigger factor of the consecutive metabolic disturbances. It had been shown, that low vitamin D level may be involved in the activation of a pro-inflammatory, pro-diabetic and atherogenic pathways in obese children [2].

The presented study is an attempt to compare selected parameters of the metabolic assessment in obese patients with hypovitaminosis D and obese with normal 25OHD levels. The study is also the first attempt to assess a possible relationship between FGF23, FGF19 and vitamin D status in obese adolescents. 


\section{OBJECTIVE}

The aim of the study is to determine the possible relationship between hypovitaminosis $\mathrm{D}$ and specific metabolic consequences of obesity (lipid disorders, non-alcoholic liver steatosis and arterial hypertension) in adolescents. Additionally, determination of a possible relationship between FGF23, FGF19 and vitamin D status in obese adolescents was investigated.

\section{MATERIALS AND NETHOD}

The study included 30 obese adolescents (14 male and 16 female) at the age of pubertly (mean age: 13.23 years, $95 \% \mathrm{CI}$ 12.64-13.8).

Body weight and height were measured to the nearest $0.1 \mathrm{~kg}$ and $0.1 \mathrm{~cm}$, respectively, using a stadiometer (Harpenden, UK) and a balance scale. As the standard of references for normal BMI SDS, values from the local population were used. Pubertal development was assessed according to the Tanner scale - Tanner stage IV or V.

Systolic (SBP) and diastolic (DBP) blood pressure were measured 3 times, every 3 minutes, using a pneumatic sphygmomanometer. Mean values were counted from obtained measures. Arterial hypertension (HA) was defined as mean SBP and/or DBP over 95th percentile for age, height and gender.

Fasting levels of 25OHD were measured by highperformance liquid chromatography. Hypovitaminosis D was defined as $25 \mathrm{OHD}<20 \mathrm{ng} / \mathrm{mL}(50 \mathrm{nmol} / \mathrm{L})$. Fasting concentration levels of glucose, total cholesterol LDL cholesterol (LDLc); HDL cholesterol concentration (HDLc), triglycerides (Tgl), uric acid (UA), alanine aminotransferase (ALT), asparginian aminotransferase (AST), gammaglutamylo transferase (GGT), calcium and phosphorus, were estimated in the fasting blood sample by the dry chemistry method with a Vitros 5.1.FF machine. Insulin concentrations were measured with immunoradiometric kits (BioSource Company Europe SA). HOMA IR was calculated using the formula: [fasting insulin level $(\mu \mathrm{IU} / \mathrm{mL}) \mathrm{x}$ fasting glucose level (mmol/L)]/22.5. Serum FGF23 levels were measured in a fasting blood sample by Human Intact FGF23 EnzymeLinked ImmunoSorbent Assay (Immunotopics Inc. USA), a two affinity purified goat polyclonal antibodies to detect epitopes with the amino-terminal and the carboxyterminal portions of FGF23. Serum FGF19 levels were measured in a fasting blood sample by Human FGF19 Enzyme-Linked Immuno Sorbent Assay (ELISA) (BioVendor - Laboratorni medicina a.s., Czech Republic).

Ultrasonography was performed using Philips EnVisor unit with an $3.5 \mathrm{MHz}$ scanhead.

Statistical analysis. To compare two sets of data, the t-Student test was employed for independent samples, and in the case of absence of normal data distribution - the two-sided MannWhitney $U$ test was used. The level of significance was set at $\mathrm{p}<0.05$. Calculations were performed using STATISTICA 10.0 PL.

The study was conducted according to the principles expressed in the Declaration of Helsinki, and approved by the Jagiellonian University Bioethical Committee (Decision No. KBET/38/B/2008). All participants and their parents signed informed consent.

\section{RESULTS}

Mean BMI value in the whole group was $32.5 \mathrm{~kg} / \mathrm{m} 2$ [SD 4.85]; mean BMI SDS - 4.65 [SD 1.67]. There was no significant difference concerning BMI values in patients with normal and low $25 \mathrm{OHD}$ levels $-30.5 \mathrm{~kg} / \mathrm{m} 2$ [SD 4.02 ] vs $33.8 \mathrm{~kg} / \mathrm{m} 2$ [SD 5.12], and also BMI SDS values 4.07 [SD 1.3] vs 5.03 [SD 1.8] respectively (Table 1 ).

Table 1. Comparison of selected parameters in the metabolic assessment of obese patients with hypovitaminosis $D$, and obese patients with normal 25OHD levels

\begin{tabular}{lccc}
\hline Parameters & $\begin{array}{c}\text { Hypovitaminosis } \mathrm{D} \\
\mathrm{n}=18 \\
\text { mean }(\mathrm{SD})\end{array}$ & $\begin{array}{c}\text { Normal vitamin } \\
\mathrm{D} \text { level } \\
\mathrm{n}=12 \\
\text { mean (SD) }\end{array}$ & p value \\
\hline Age [years] & $13.7(1.25)$ & $12.6(1.86)$ & 0.09 \\
\hline BMI SDS & $5.03(1.8)$ & $4.07(1.3)$ & 0.16 \\
\hline SBP [mmHg] & $125.9(11.1)$ & $115(15)$ & 0.07 \\
\hline DBP [mmHg] & $78.6(11.5)$ & $69(10.1)$ & $\mathbf{0 . 0 4}$ \\
\hline fasting glucose [mmol/L] & $4.56(0.48)$ & $4.57(0.57)$ & 0.21 \\
\hline fasting insulin [IU/L] & $21.9(9.9)$ & $18.1(5.1)$ & 0.5 \\
\hline HOMA IR & $4.5(2.3)$ & $3.8(1.1)$ & 0.66 \\
\hline Total cholesterol [mmol/L] & $4.32(0.8)$ & $4.7(0.9)$ & 0.37 \\
\hline LDLc [mmol/L] & $2.6(0.7)$ & $2.9(0.9)$ & 0.34 \\
\hline HDLc [mmol/L] & $1.1(0.2)$ & $1.2(0.4)$ & 0.98 \\
\hline Triglicerydes [mmol/L] & $1.3(0.54)$ & $1.5(0.64)$ & 0.4 \\
\hline Uric acid [umol/L] & $384.7(66.8)$ & $301.5(80.7)$ & $\mathbf{0 . 0 1 *}$ \\
\hline AST [IU/L] & $26.7(8.6)$ & $25.2(5.1)$ & 0.88 \\
\hline ALT [IU/L] & $34.8(19.7)$ & $29.3(5.1)$ & 0.5 \\
\hline GGT [IU/L] & $27.1(2.8)$ & $18(5.3)$ & 0.9 \\
\hline Ca [mmol/L] & $2.43(0.14)$ & $2.47(0.07)$ & 0.35 \\
\hline P[mmol/L] & $1.4(0.25)$ & $1.65(0.11)$ & $\mathbf{0 . 0 4 *}$ \\
\hline FGF23 [pg/mL] & $6.08(2.83)$ & $7.11(2.88)$ & 0.47 \\
\hline FGF19[pg/mL] & $128.1(65)$ & $150(15)$ & 0.39 \\
\hline
\end{tabular}

The mean 25OHD level was $19.78 \mathrm{ng} / \mathrm{mL}$ (95\%CI 15.923.6). Hypovitaminosis $\mathrm{D}$ was recognized in 18 patients $(60 \%$; 10 girls and 8 boys). The vitamin D deficient group presented with significantly higher values of diastolic blood pressure (125.9 vs. $115 \mathrm{mmHg}$; $=0.04)$, uric acid level (384.7 umol/L vs. $301.5 \mathrm{umol} / \mathrm{L} ; \mathrm{p}=0.01)$, and lower phosphorus level (1.4 vs. $1.65 \mathrm{mmol} / \mathrm{L} ; \mathrm{p}=0.04)$. In patients with hypovitaminosis D mean values of: HOMA IR (4.5 vs. 3.8; $\mathrm{p}=0.66)$, ALT (34.8 vs. $29.3 \mathrm{IU} / \mathrm{L} ; \mathrm{p}=0.5)$ and GGT (27.1 vs. $18 \mathrm{IU} / \mathrm{L} ; \mathrm{p}=0.9)$, were higher, but the differences were not significant. Further analysis showed lower, mean FGF23 and FGF19 levels (6.08 vs.7.11; $\mathrm{p}=0.47 ; 128.1$ vs. $150 ; \mathrm{p}=0.39$, respectively), but the differences were not significant. Detail comparison of the mean values of the selected biochemical parameters and blood pressure are shown in Table 1. More than 44\% (8 of 18) of patients with hypovitaminosis D presented with arterial hypertension, while only one patient with normal vitamin D level had elevated SBP. Liver steatosis features in the ultrasound examination and was present in over $44 \%$ (8 of 18 ) of the patients with low, and in $25 \%$ (3 of 12) of the patients with normal vitamin $\mathrm{D}$ level. 


\section{DISCUSSION}

Vitamin D deficiency, defined as serum concentration less than $20 \mathrm{ng} / \mathrm{mL}$, is one of the most common nutritionresponsive medical conditions worldwide $[1,2,3]$. However, although the association between hypovitaminosis $\mathrm{D}$ and obesity is well-established, there is still uncertainty which one is the cause, and which one is a consequence, and what the health consequences of lower concentrations of vitamin $\mathrm{D}$ might be. In the literature, the suggested mechanisms associated with obesity include: low calcium and vitamin D intake, less skin exposure to the sun due to reduced outdoor activity, reduced activation and/or increased catabolism than non-obese individuals, and sequestration of $25 \mathrm{OHD}$ in adipose tissue [3]. In children and adolescents who are overweight or obese it affects approximately $60 \%$ in comparison to $20 \%$ of non-obese subjects $[1,2,3]$. However, an inverse correlation between BMI, fat tissue content and vitamin $\mathrm{D}$ levels had been proved in many studies, and a recently published metaanalysis of 21 adult cohorts (up to 42,024 participants) revealed that higher BMI lead to lower vitamin D level; but in contradiction, low vitamin D level does not contribute to the increase in the BMI [3]. These results confirm that hypovitaminosis D is rather a consequence, not a cause of obesity. Epidemiologicalpooled analysis of prospective observational studies of diverse populations demonstrates that hypovitaminosis D is associated with a slight risk of cardiovascular events [4, $5,6,7]$. The decrease of the vitamin $\mathrm{D}$ level has also been associated with higher blood pressure levels, as already shown in many prospective studies, as well as meta-analyses of observational studies $[5,6]$. The results of the presented study confirm that both systolic and diastolic blood pressure in patients with hypovitaminosis $\mathrm{D}$ were higher, and for the diastolic blood pressure the difference was significant (125.9 vs. $115 \mathrm{mmHg}$; $=0.04$ ). Moreover, the incidence of arterial hypertension among obese patients with vitamin D deficiency was higher than in peers with normal vitamin $D$ level (44\% vs. $8.3 \%$ ). Low vitamin D level was also associated with an elevated uric acid concentration level. An association between vitamin $\mathrm{D}$ deficiency and hyperuricemia in obese adolescents has not been reported; however, a significant association between vitamin D insufficiency and elevated uric acid was recently found in postmenopausal women [8]. This novel observation seems to be particularly interesting since it had been proved that an elevated uric acid level is a significant and independent predictor of the development of the arterial hypertension, especially in young individuals, and metabolic syndrome in the future $[9,10]$. These findings may suggest that low vitamin D level is not only one of the complications of the obesity, but it can be involved in the development of other metabolic complications. The relationship between low vitamin D and elevated uric acid, and subsequent cardiovascular complications, is currently the subject of many studies. The results of the studies published to date allow only speculation about the potential mechanism and need confirmation by the future investigations. It is suggested that vitamin D deficiency or decreased bioactivity can activate parathyroids to induce the release of parathyroid hormone (PTH). That, in turn, is considered to raise the uric acid level, but details of this mechanism remain unknown [9, $10,11]$. It is suspected that elevation of the uric acid might be associated with the fluctuations of the fibroblast growth factor
23 (FGF23) level [12, 13, 14]. FGF23 is a protein synthesized by osteocytes that has been described as having a key role in the 'bone-kidney-parathyroid' axis and the regulation of phosphate-calcium-PTH and 1.25(OH)D metabolism. Recent studies have pointed to the potential role of the FGF23 in the development of insulin resistance and its metabolic consequences. Previous research by the authos of the current has shown that FGF23 may play a key role in the early phase of decreased insulin sensitivity in obese adolescents $[15,16]$.

The results of the presented study show, that in young patients with obesity and hypovitaminosis $\mathrm{D}$, without clinical signs or symptoms of kidney disease, the FGF23 levels were lower in comparison to individuals with normal 25OHD levels; however, the difference was not statistically significant. Interestingly, the group with low vitamin D level and lower FGF23 level presented with significantly higher concentration levels of uric acid. This observation does not confirm the results of the previously mentioned study performed in pediatric patients with chronic kidney disease, and preserved kidney function [13], and points to a different and more complex mechanisms in adolescents with obesity. In obese adolescents, a low vitamin $\mathrm{D}$ level may slightly increase PTH which, in turn, can activate 1 alpha-hydroxylase and subsequently increase the 1,25OHD level, which $t$ may be a potential cause of the further decrease of the 25OHD level, leading to a vicious circle. Moreover, a moderate rise of the PTH level may decrease the phosphorus level, which is associated with the slight decrease of the FGF23 level, as shown in previous studies conducted in populations with normal kidney function [17].

The results of the presented study show higher, but not significant, values of the HOMA IR in patients with hypovitaminosis $\mathrm{D}$ (4.5 vs. $3.8, \mathrm{p}=0.66)$. However, some larger studies confirmed the relationship between vitamin $\mathrm{D}$ deficiency and insulin resistance $[19,20]$. Since FGF23 is strongly involved in calcium-phosphorus-vitamin D metabolic pathways, it is reasonable to suspect that its contribution to the development of insulin resistance may be related to vitamin $\mathrm{D}$ deficiency, which is also considered an insulin resistance risk factor. Moreover, recent studies conducted on mice indicate that insulin signaling, or fat metabolism disturbances in the genetically ablated FGF23 are mediated by vitamin $\mathrm{D}[19,20,21]$. Some studies have pointed to the presence of low FGF23 levels in vitamin D deficiency; but this was not confirmed in larger groups [22]. These insights indicate the potential contribution of vitamin $\mathrm{D}$ to the development of insulin resistance in a novel way. The presented study is the first attempt to assess a possible relationship between FGF23 and vitamin D status in obese adolescents.

Another interesting observation, although also not statistically significant, is the elevation of liver enzymes levels, especially ALT and GGT, in patients with hypovitaminosis D ( 34.8 vs. $29.3 \mathrm{IU} / \mathrm{L} ; \mathrm{p}=0.5 ; 27.1$ vs. $18 \mathrm{IU} /$; $\mathrm{p}=0.9$ respectively). The ultrasound revealed liver steatosis in $44.4 \%$ of the patients with low, and in $25 \%$ with normal vitamin D levels. That confirms the results of the meta-analysis published recently by Eliades et. al., that revealed significantly decreased serum 25OHD concentrations in patients with non-alcoholic fatty liver disease (NAFLD), suggesting that vitamin D may play a role in the development of this condition [21]. On the other side, as native vitamin $\mathrm{D}$ is converted to $25 \mathrm{OHD}$ in the liver, it remains controversial whether low $25 \mathrm{OHD}$ level is a cause 
or a consequence of liver function impairment. Nevertheless, additional analysis performed in our study showed lower concentration level of the fibroblast growth factor 19 (FGF19) in patients with hypovitaminosis D. Fibroblast growth factor 19 (FGF19) is a hormone released from the small intestine; recently, it has emerged as an endocrine regulator of glucose and lipid metabolism. The results of the presented study, although not statistically significant, need particular attention, since a decrease in fasting FGF19 is an independent risk factor for the development of NAFLD in obese adolescents $[23,24,25]$. Its relationship to vitamin D deficiency needs further investigation.

Although the group investigated in the presented study was relatively small, the very interesting and promising preliminary results obtained indicate the possible contribution of vitamin D deficiency in the development of metabolic obesity complications. The mechanisms of such contribution need further investigation.

\section{CONCLUSIONS}

Hypovitaminosis D in obese adolescents is associated with a higher prevalence of arterial hypertension, liver steatosis, elevated serum uric acid and low phosphorus levels. The potential contribution of the fibroblast growth factor 23 and fibroblast growth factor 19 in the development of these complications needs further investigation.

\section{REFERENCES}

1. Creo AL, Rosen JS, Ariza AJ, et al. Vitamin D levels, insulin resistance, and cardiovascular risks in very young obese children. J Pediatr Endocrinol Metab. 2013; 26: 97-104.

2. Reyman M, Verrijn Stuart AA, van Summeren M, et al. Vitamin $\mathrm{D}$ deficiency in childhood obesity is associated with high levels of circulating inflammatory mediators, and low insulin sensitivity. Int J Obes (Lond). 2013; doi: 10.1038/ijo.2013.75.

3. Vanlint S. Vitamin D and Obesity Nutrients 2013; 5, 949-956.

4. Ku YC, Liu ME, Ku CS, Liu TY, Lin SL. Relationship between vitamin D deficiency and cardiovascular disease. World J Cardiol. 2013; 5: 337-346.

5. Burgaz A, Orsini N, Larsson SC, Wolk A. Blood 25-hydroxyvitamin D concentration and hypertension: A meta-analysis. J Hypertens. 2011; 29: 636-645.

6. Pilz S, Tomaschitz A. Role of vitamin D in arterial hypertension. Expert Rev Cardiovasc Ther. 2010; 8: 1599-1608.

7. Kienreich K, Tomaschitz A, Verheyen N, et al. Vitamin D and cardiovascular disease. Nutrients. 2013; 5: 3005-21.
8. Peng H, Li H, Li C, et al. Association between vitamin D insufficiency and elevated serum uric acid among middle-aged and elderly Chinese Han women. PLoS One. 2013; 8: e61159.

9. Nagahama K, Inoue T, Kohagura K, et al. Hyperuricemia predicts future metabolic syndrome: a 4-year follow-up study of a large screened cohort in Okinawa, Japan. Hypertens Res. 2013; 31. doi: 10,1038/hr.2013,137

10. Grayson PC, Kim SY, LaValley M, Choi HK. Hyperuricemia and incident hypertension: A systemic review and meta-analysis. Arthritis Care Res. 2011; 63(1): 102-110.

11. Hui JY, Choi JW, Mount DB, Zhu Y, Zhang Y, et al. The independent association between parathyroid hormone levels and hyperuricemia: a national population study. Arthritis Res Ther. 2012; 14: R56.

12. Bacchetta J, Cochat P, Salusky IB, Wesseling-Perry K. Uric acid and IGF1 as possible determinants of FGF23 metabolism in children with normal renal function. Pediatr Nephrol. 2012; 27: 1131-8.

13. Wolf M. Update on fibroblast growth factor 23 in chronic kidney disease. Kidney Int. 2012; 82: 737-47.

14. Gutiérrez OM, Wolf M, Taylor EN. Fibroblast growth factor 23, cardiovascular disease risk factors, and phosphorus intake in the health professionals follow-up study. Clin J Am Soc Nephrol. 2011; 6: 2871-8.

15. Wojcik M, Janus D, Dolezal-Oltarzewska K et al. The association of FGF23 levels in obese adolescents with insulin sensitivity. J Pediatr Endocrinol Metab. 2012; 25: 687-690.

16. Wojcik M, Dolezal-Oltarzewska K, Janus D, et al. FGF23 contributes to insulin sensitivity in obese adolescents - preliminary results. Clin Endocrinol. 2012; 77: 537-540.

17. Liu S, Tang W, Zhou J, et al. Fibroblast growth factor 23 is a counterregulatory phosphaturic hormone for vitamin D. J Am Soc Nephrol 2006; 17:1305-1315.

18. Dai B, David V, Martin A, et al. A comparative transcriptome analysis identifying FGF23 regulated genes in the kidney of a mouse CKD model. PLoS One. 2012; 7:e44161.

19. Forouhi NG, Luan J, Cooper A, Boucher BJ, Wareham NJ. Baseline serum 25-hydroxy vitamin $\mathrm{d}$ is predictive of future glycemic status and insulin resistance the medical research councilely prospective study 1990-2000. Diabetes. 2008; 57(10): 2619-2625.

20. Kelly A, Brooks LJ, Dougherty S, Carlow DC, Zemel BS. A crosssectional study of vitamin D and insulin resistance in children. Arch Dis Childhood. 2011; 96(5): 447-452.

21. Streicher C, Zeitz U, Andrukhova O, et al. Long-term Fgf23 deficiency does not influence aging, glucose homeostasis, or fat metabolism in mice with a nonfunctioning vitamin D receptor. Endocrinology. 2012; 153: $1795-1805$.

22. Razzaque MS, Sitara D, Taguchi T, et al. Premature aging-like phenotype in fibroblast growth factor 23 null mice is a vitamin D-mediated process. FASEB J. 2006; 20: 720- 722.

23. Dai B, David V, Martin A, et al. A comparative transcriptome analysis identifying FGF23 regulated genes in the kidney of a mouse CKD model. PLoS One. 2012; 7:e44161.

24. Eliades M, Spyrou E, Agrawal N, et al. Meta-analysis: vitamin D and non-alcoholic fatty liver disease. Aliment Pharmacol Ther. 2013; 38(3): 246-54.

25. Wojcik M, Janus D, Dolezal-Oltarzewska K, et al. A decrease in fasting FGF19 levels is associated with the development of non-alcoholic fatty liver disease in obese adolescents. J Pediatr Endocrinol Metab. 2012; 25(11-12): 1089-93. 